were 22 cases in Group non-aspiration, 4 cases received direct stenting, 18 cases received balloon predilatation and stenting. 5 cases showed slow flow, 3 cases recovered normal flow after intra-coronary infusion of nitroglycerin, verapamil and Xinweinin, but 2 cases also showed TIMI 1, and accompanied heart failure.

Conclusions The applying of aspiration catheter in patients with STEMI prior to primary PCI could increase the opportunities of direct stenting, improve myocardial reperfusion, immediate and recent clinical outcomes as compared with PCI in the absence of thrombus aspiration, and also show ease and safe procedure.

\section{e0420 C-REACTIVE PROTEIN FOR PREDICTING CLINICAL OUTCOMES AFTER DRUG-ELUTING STENT IMPLANTATION}

doi:10.1136/hrt.2010.208967.420

Yu Miao, Zhou Yujie, Wang Zhijian, Shi Dongmei, Liu Yuyang, Zhao Yingxin, Guo Yonghe. Anzhen Hospital Capital Medical University

Background Although C-reactive protein (CRP) has been proposed as a useful biomarker for predicting atherothrombosis, the association of CRP levels and clinical outcomes after drug-eluting stent (DES) implantation has not been defined.

Objectives To assess the predictive value of CRP after drug-eluting stent (DES) implantation, especially the association of CRP levels with adverse clinical events, such as stent thrombosis, death, myocardial infarction (MI) and so on.

Methods We evaluated 2048 consecutive patients who underwent successful DES implantation. The primary outcomes were stent thrombosis, death, myocardial infarction (MI), and target vessel revascularisation.

Results After 2 years of follow-up, there were 11 stent thrombosis, 72 deaths, 113 MI, 133TVR. In multivariable Cox proportionalhazards models, the high CRP remained predictive of adverse cardiac events, elevated levels of CRP were significantly associated with increased risks of stent thrombosis (HR 4.08; 95\% CI 1.91 to 11.44; $\mathrm{p}<0.001$ ), death (HR 2.01; 95\% CI 1.18 to 4.688; $\mathrm{p}=0.004$ ), MI (HR 1.78 ; $95 \%$ CI 1.25 to $9.12 ; \mathrm{p}=0.001)$, but not target vessel revascularisation (HR 1.20; 95\% CI 0.59 to 1.16; $\mathrm{p}=0.62$ ).

Conclusions Elevated CRP levels were significantly associated with major coronary events after DES implantation, such as stent thrombosis, death, and MI, demonstrating the additive impacts of active inflammation and myocardial injury on prognosis after DES implantation.

\section{e0421 DIAGNOSTIC VALUE OF PLASMA SRAGE IN CORONARY ARTERY DISEASE AND CORONARY ARTERY DISEASE WITH ACUTE MYOCARDIAL INFARCTION (AMI)}

doi:10.1136/hrt.2010.208967.421

${ }^{1}$ Guo Caixia, 'Zhu Xinyuan, ${ }^{2}$ Zhang Like, ${ }^{1}$ Song Ke, ${ }^{1}$ Xu Xiaowei, ${ }^{1} \mathrm{Du}$ Fenghe. ${ }^{1}$ Department of Cardiology Medicine, Beijing Tiantan Hospital Affiliate of Capital University of Medical Sciences, Beijing, China; ${ }^{2}$ Department of Pathophysiology, Capital University of Medical Sciences, Beijing, China

Objective To elucidate whether plasma soluble receptor for advanced glycation end products (sRAGE) is the biochemical markers indicating coronary artery disease and coronary artery disease with acute myocardial infarction (AMI).

Method Plasma levels of sRAGE was determined by enzyme linked immunoabsorbent assay in patients who came from Cardiology Department of Tiantan Hospital form March to May 2009 categorised as group I (non-CAD subjects), group II (CAD without myocardial infarction subjects), and group III (CAD with AMI subjects).

Results Plasma levels of sRAGE was higher in group III than in group II $(p<0.01)$ and in group I $(p<0.01)$. The levels of sRAGE between group II and group I were of no statistical difference $(p>0.05)$. Further more, to evaluate whether sRAGE is the biochemical markers indicating the AMI in CAD patients, ROC curve was used, and area under the curve was $0.855(\mathrm{p}=0.000)$.

Conclusion sRAGE may be new biochemical markers indicating AMI in the patients with coronary artery disease. The diagnostic sensitivity of sRAGE is $75 \%$ and specificity is $86.4 \%$.

\section{e0422 DIAGNOSTIC VALUE OF ADENOSINE STRESS ${ }^{\text {99M}}$ TC-MIBI GATED MYOCARDIAL PERFUSION IMAGING FOR CORONARY ARTERY DISEASE}

doi:10.1136/hrt.2010.208967.422

${ }^{1}$ Wang Lijuan, ${ }^{1} \mathrm{Li}$ Xiaojuan, ${ }^{1}$ Sun Yingxian, ${ }^{2} \mathrm{Li} \mathrm{Na},{ }^{2} \mathrm{Li}$ Yaming. ${ }^{1}$ Department of Cardiology The First Affiliated Hospital of China Medical University Shenyang, China; ${ }^{2}$ Department of Nuclear Medicine The First Affiliated Hospital of China Medical University Shenyang, China

Objective Stress ${ }^{99 \mathrm{~m}}$ Tc-MIBI myocardial perfusion imaging (MPI)) be useful in evaluating myocardial ischaemia and judging coronary artery stenosis. The purpose of this study was to evaluate the sensitivity, specificity and accuracy of adenosine stress ${ }^{99 \mathrm{~m}} \mathrm{Tc}-\mathrm{MIBI}$ gated myocardial perfusion SPECT (G-MPI) for the diagnosis of coronary artery disease (CAD).

Materials and methods The subjects were forty-six patients diagnosed or suspected $\mathrm{CAD}$, including forty with angina, six with old myocardial infarction. Adenosine stress G-MPI were performed 90 min after injection of adenosine, and resting G-MPI performed in same day. All patients underwent coronary artery angiography within two weeks. Significant stenosis was defined when the coronary artery intraluminal stenosis $\geq 50 \%$. Analyse the imaging and calculate the sensitivity, specificity and accuracy of adenosine stress G-MPI in diagnosing CAD and judging coronary artery stenosis. Do correlation analysis of left ventricular systolic function (left ventricular ejection fraction) between adenosine stress ${ }^{99 m}$ Tc-MIBI G-MPI and echocardiography.

Results The sensitivity, specificity, accuracy, positive predictive value and negative predictive value of adenosine stress ${ }^{99 \mathrm{~m}} \mathrm{Tc}-\mathrm{MIBI}$ G-MPI for the diagnosis of CAD were $87.0 \%, 85.7 \%, 86.6 \%, 93.8 \%$, and $72.7 \%$ respectively. The sensitivity, specificity and accuracy for the diagnosis of LAD, LCX and RCA stenosis were $88.2 \%, 90.9 \%$, $88.9 \% ; 78.6 \%, 83.3 \%, 80.0 \% ; 90.5 \%, 81.8 \%, 87.5 \%$ respectively. LVEF-G-MPI correlated with LVEF-UCG significantly, with a correlation coefficient of $0.885(\mathrm{R}=0.0001)$.

Conclusions In Conclusion, stress adenosine ${ }^{99 \mathrm{~m}} \mathrm{Tc}-\mathrm{MIBI}$ G-MPI have provided better sensitivity, specificity and accuracy in the diagnosis of $\mathrm{CAD}$, and is probably an accurate method for detecting coronary artery branch stenosis. It can be used in evaluating left ventricular function, especially for patients unsuited in the exercise MPI.

\section{e0423 THE RESEARCH ABOUT THE SHORT-TIME EFFECTS OF PREINFRACTION ANGINA IN PATIENTS COMPLICATED WITH ACUTE MYOCARDIAL INFRACTION AND TREATED BY PRIMARY PERCUTANEOUS CORONARY INTERVENTION}

doi:10.1136/hrt.2010.208967.423

Cao Rui Caoxuebin. 252 Hospital of Baoding, Hebei, China

Objective To evaluate the short-time effects, angiographic characteristics, and the clinical outcome of preinfarction angina in patients with first acute myocardial infarction (AMI) after emergency primary percutaneous coronary intervention (PCI). And evaluate the influence of diabetes mellitus to preinfarction angina.

Methods The clinical data of 130 patients with AMI hospitalised from January 2006 to December 2008, who underwent emergency 